\title{
L'absence de préconception du temps chez Épicure
}

Lecture de la Lettre à Hérodote, 72-73

\section{Marianne Gœury}

\section{OpenEdition}

Journals

Édition électronique

URL : https://journals.openedition.org/philosant/934

DOI : $10.4000 /$ philosant.934

ISSN : 2648-2789

Éditeur

Éditions Vrin

\section{Édition imprimée}

Date de publication : 1 novembre 2012

Pagination : 89-114

ISBN : 978-2-7574-0400-3

ISSN : 1634-4561

Référence électronique

Marianne Gœury, "L'absence de préconception du temps chez Épicure », Philosophie antique [En ligne], 12 | 2012, mis en ligne le 01 novembre 2018, consulté le 02 décembre 2022. URL : http:// journals.openedition.org/philosant/934 ; DOI : https://doi.org/10.4000/philosant.934

\section{(c)}

Creative Commons - Attribution - Pas d'Utilisation Commerciale - Pas de Modification 4.0 International - CC BY-NC-ND 4.0

https://creativecommons.org/licenses/by-nc-nd/4.0/ 


\section{L'ABSENCE DE PRÉCONCEPTION DU TEMPS CHEZ ÉPICURE. \\ Lecture de la Lettre à Hérodote, 72-73 \\ Marianne GOEURY \\ Académie de Créteil}

RÉSUMÉ. Le passage de la Lettre à Hérodote consacré au temps (Hrdt. 72-73) est particulièrement obscur. Épicure y affirme explicitement que le mot temps ne renvoie à aucune préconception (prolepse), en tout cas pas au sens habituel qu'a ce terme pour lui, puisqu'il n'existe aucun contenu commun à toutes nos expériences du temps; et cependant le mot temps n'est pas vide. Cet article vise à résoudre ce paradoxe. Il éclaircit la nature de la perception du temps épicurien et tente de reconstituer le processus cognitif qui la sous-tend. La perception du temps est idiosyncrasique, car elle porte sur une entité de troisième degré : le temps est en effet, selon la formule attribuée à Épicure par Démétrius Lacon, un «accident d'accidents $\gg$. Sa perception implique une combinaison d'intuition et de déduction, d'analogie et d'épilogisme, en deçà de la formation d'une préconception - dont nous expliquons l'impossibilité. Elle concerne toujours les durées en tant que nous les rapportons les unes aux autres, selon un « libre jeu » de l'épilogisme et de l'analogie qui ne donne pas lieu à une préconception, mais rend possible de nommer le temps.

SUMMARY. The passage from the Letter to Herodotus devoted to the topic of time (Hrdt. 72-73) is particularly obscure. Epicurus states that the word time is not associated with any preconception (prolepsis), not anyway as he usually conceives of it, since there is no content shared by all our experiences of time; however, the word time is not empty. The purpose of this paper is to solve this paradox. It clarifies the nature of the perception of Epicurean time, and tries to reconstruct the cognitive process which underlies it. The perception of time is indeed idiosyncrasic, since its object is a thirddegree entity: Epicurean time is, according to Demetrius Laco, an "accident of accidents". Its perception implies a combination of analogy and epilogism, which is not followed by the formation of a preconception - we explain in the paper why it is impossible. It always pertains to the durations insofar as we relate them to each other, through a "free play" between analogy and epilogism which does not produce a preconception, but makes it possible to name time. 



\section{Introduction}

Aux paragraphes 72-73 de la Lettre à Hérodote, immédiatement après avoir évoqué sa théorie des accidents, Épicure évoque le thème du temps, en quelques lignes singulièrement peu claires. En effet, s'il en ressort que le temps a un statut exceptionnel au regard de la canonique épicurienne, le diagnostic précis de cette exceptionnalité n'est pas facile à établir, et les commentateurs dépassent rarement le simple constat de l'obscurité du passage ${ }^{1}$. Tous s'accordent cependant sur le fait que le mot temps ne renvoie pas à une préconception, ou du moins pas à une préconception au sens classique du terme. Du temps, écrit en effet Épicure, nous ne connaissons que « l'évidence selon laquelle nous parlons de "temps long" ou de "temps court" », et le temps se réduit à « un certain accident particulier, en référence auquel nous utilisons le mot temps $s^{3} \gg$, contrairement « à tout ce que nous recherchons dans un substrat, et que nous rapportons aux prénotions considérées en nous-mêmes $\gg{ }^{4}$. Or tout mot doit, selon la canonique épicurienne, renvoyer à une préconception ou à une notion commune, formées à même les sensations ou obtenues à l'issue d'un raisonnement lui-même élaboré à partir de celles-ci ${ }^{5}$; et les mots qui n'obéissent pas à cette règle sont qualifiés par Épicure de « sons vocaux vides $^{6} \gg$. Le mot temps, naturellement utilisé par tous, ne saurait cependant être considéré de la sorte : il est donc à la fois légitime et dépourvu de contenu énonçable. Pour être légitime, il doit désigner quelque chose de commun aux expériences que nous faisons à chaque fois que nous parlons en termes temporels; mais nous sommes incapables de donner une

1. Morel 2002, p. 195, note ainsi que ce n'est pas seulement l'expression, comme l'affirme Cyril Bailey, mais aussi l'idée générale, qui est « loin d'être transparente ».

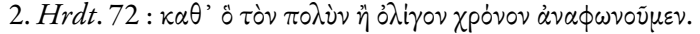

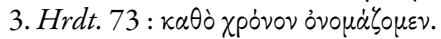

4. Hrdt. 72 (trad. Balaudé 1999). Sauf indication contraire, c'est cette traduction qui est utilisée dans la suite.

5. Cf. Hrdt. 38 : « Car il est nécessaire que, pour chaque son vocal, la notion première soit vue et n'ait nullement besoin de démonstration $[\ldots] \gg$. Voir également Sextus Empiricus, $M$ I, 57 (255 Us.) et Diogène Laërce, $\mathrm{X}, 33$.

6. Hrdt. 37. 
formulation de ce noyau commun de toutes nos perceptions temporelles. Pierre-Marie Morel, dans son article sur le temps épicurien ${ }^{7}$, suggère que, par «notion première », en $H r d t .38^{8}$, Épicure entende une notion plus large que la préconception, de sorte que le temps ne ferait pas exception à l'association de tout mot à une notion première, puisque nous aurions

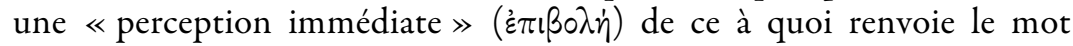
temps, à laquelle il suffit de se référer pour toute enquête sur le temps. Il nous semble au contraire que les paragraphes $72-73$ de la Lettre à Hérodote tendent précisément à montrer que nous n'avons pas une perception immédiate $d u$ temps, et que le mot temps renvoie seulement à une pluralité irréductible de perceptions (celles des différentes durées), ce qui explique l'absence d'une préconception - ou même d'une notion première - du temps : ce sera le premier point de notre analyse.

Un tel constat laisse ouverte la question de la nature de ce qui est sousjacent au mot temps. Comment éclairer ce paradoxe d'un terme dépourvu de contenu immédiatement assignable, et cependant pourvu de sens ? Il faut pour cela comprendre le raisonnement qu'Épicure engage son lecteur à effectuer pour saisir le contenu du mot temps; il s'agit d'une reconstitution du processus cognitif mi-intuitif, mi-déductif activé lors de chacune de nos perceptions du temps, et qui consiste en une combinaison d'analogie et d'épilogisme. Après avoir tenté d'en restituer les étapes, nous suggérerons que l'on en reste, dans le cas du temps, au stade de la genèse du processus qui mène habituellement à la formation d'une préconception; ce processus, qui s'accompagne d'évidence à chaque occurrence d'une durée, reste en effet ouvert et n'aboutit jamais à la saisie d'un terme général qui viendrait à la fois le résumer et le clore. Le mot temps renvoie ainsi à une procédure cognitive de troisième degré - le temps est en effet pour Épicure, selon Démétrius Lacon, un « accident d'accidents ${ }^{9}$ » - qui vient se superposer à la perception des autres phénomènes et qui constitue ainsi en quelque sorte, pour utiliser un terme anachronique, une forme de l'expérience - même si le temps, comme l'âme, fait bien évidemment partie de la physique. Aussi quiconque s'interroge sur le temps doitil faire un effort intellectuel pour prendre conscience du processus perceptif qui se produit en lui à chaque évaluation de durée. Épicure, en Hrdt. 72-73, ne donne que des indices de ce mécanisme, que nous essaierons de reconstituer : ce sera le second point de notre argumenta-

7. Morel 2002.

8. Cf. n. 5 ci-dessus.

9. La formule est attribuée à Épicure par Démétrius Lacon selon Sextus Empiricus (MX, 219; HP III, 317) et Aétius (I, 22, 5) : «Épicure dit qu'il (scil. le temps) est un accident $<$ d'accidents $>$, c'est-à-dire quelque chose qui accompagne les mouvements. » 
tion. La mise en évidence de ce mécanisme permettra d'expliquer pourquoi, si nous ne disposons d'aucune préconception ou notion première du temps, nous restons cependant capables de parler « du » temps.

\section{L'exclusion d'une préconception du temps}

Les deux règles fondamentales de la méthode épicurienne sont formulées en $H r d t$. 37-38: la première engage à « saisir ce qui est placé sous les sons vocaux », et la seconde à «observer toutes choses suivant les sensations, et en général suivant les appréhensions présentes, tant celles de la pensée que celles de n'importe quel critère, et de la même façon les affections existantes $\gg$. Le cas du temps fait exception aux deux règles; à la seconde, parce qu'il échappe à la distinction entre réalités évidentes (saisies par la perception) et réalités inévidentes (déduites par la pensée); et à la première, parce qu'il n'y a pas de préconception du temps. Rappelons tout d'abord la formulation de la première règle :

Pour commencer, Hérodote, il faut saisir ce qui est placé sous les sons vocaux, afin qu'en nous y rapportant nous soyons en mesure d'introduire des distinctions dans ce qui est matière à opinion - que cela suscite une recherche ou soulève une difficulté -, et pour éviter que tout ne reste pour nous sans distinction dans des démonstrations que nous mènerions à l'infini, ou que nous n'ayons que des sons vocaux vides. Car il est nécessaire que, pour chaque son vocal, la notion première soit vue et n'ait nullement besoin de démonstration, si nous devons bien posséder l'élément auquel rapporter ce qui suscite une recherche ou soulève une difficulté, et qui est matière à opinion. ( $H r d t$. 37-38.)

Dans la première phrase, le terme í choses qui ont été placées sous ») peut être compris comme faisant référence, soit aux préconceptions (ou aux « notions premières » évoquées dans la phrase suivante), soit aux substrats concrets de l'expérience, c'està-dire aux corps. Le parallélisme entre $\mathrm{Hrdt}$. 38 et $\mathrm{Hrdt}$. 72 invite assez naturellement à choisir la première hypothèse, puisque les préconceptions sont explicitement mentionnées en $\mathrm{Hrdt}$. 72, dans une phrase négative qui reprend, presque mot pour mot, celle de $H r d t .38^{10}$. Selon cette

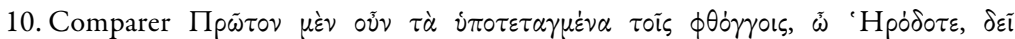

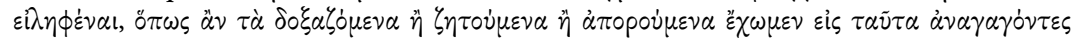

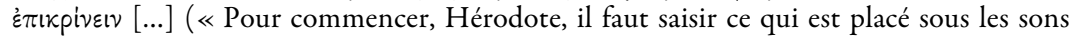
vocaux, afin qu'en nous y rapportant nous soyons en mesure d'introduire des distinctions dans ce qui est matière à opinion - que cela suscite une recherche ou soulève une

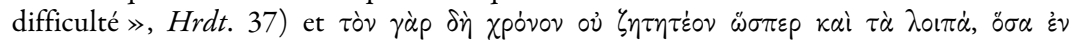

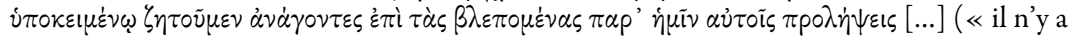
certainement pas à mener la recherche sur le temps comme sur le reste, c'est-à-dire tout ce 
lecture, la préconception est l'un des critères de vérité ${ }^{11}$. Acquise par inférence inductive à partir de similitudes observées de manière répétée, la préconception permet de saisir l'《esquisse » $\left(\tau \dot{\tau} \pi \circ \nu^{12}\right)$ de la chose, et elle est donc la condition (acquise) de toute perception, qu'elle permet d'anticiper - « pro-lepse » signifiant littéralement « saisie à l'avance ». La préconception assure ainsi l'adéquation des mots aux choses, et la possibilité de revenir aux préconceptions garantit la non-vacuité de notre discours.

Cette lecture classique de la première règle de la méthode a cependant parfois été critiquée. Une autre interprétation des í 37-38 consiste en effet à les identifier à des corps - auxquels se rapporte toujours ultimement ce qui se trouve sous les mots, puisque toute connaissance est issue, directement ou non, de la sensation. La controverse concernant la présence ou non des préconceptions en $\mathrm{Hrdt}$. 38 a des conséquences quant à l'épistémologie épicurienne : en effet, si ce n'est pas aux préconceptions qu'Épicure fait allusion en $\mathrm{Hrdt}$. 38, celles-ci ne font pas partie des éléments fondamentaux de la méthode, et ne constituent pas un critère du vrai, en tout cas pas dans l'état de la théorie épicurienne contemporain de la rédaction de la Lettre à Hérodote ${ }^{13}$. L'unique occurrence de $\pi \rho \dot{\lambda} \lambda \eta \psi \mathrm{s}$ dans la Lettre à Hérodote (celle du paragraphe 72) a ainsi été longtemps remise en cause par David Sedley, qui se fondait sur

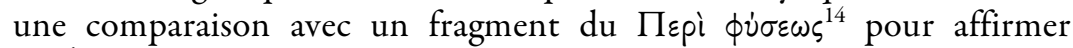
qu'Épicure, lors de la rédaction de la Lettre à Hérodote, n'avait pas encore élaboré le concept de préconception (ni celui d'épilogisme, également présent dans ce passage). Elle a aussi des conséquences concernant le temps: si la mention des préconceptions, en $\operatorname{Hrdt}$. 72, est authentique,

que nous cherchons en un substrat, et que nous rapportons aux prénotions considérées en nous-mêmes [...] », Hrdt. 72). Ainsi, pour Elizabeth Asmis (Asmis 1984, Part I, $1:$ « Epicurus' statement of the rule 》), les i் conceptions, et la première règle de la méthode épicurienne consiste à rapporter chaque mot à la préconception qui lui correspond, elle-même fondée (directement ou non) sur l'évidence empirique procurée par la sensation. Pour A. Long et D. Sedley également, « les choses sous-jacentes aux mots » sont bien les préconceptions (Cf. Long \& Sedley 2001, p. 208).

11. Le deuxième selon Diogène Laërce, entre les sensations et les affections, auxquelles il faut encore ajouter selon lui les « appréhensions imaginatives de la pensée » destinées à saisir certaines réalités plus subtiles. Voir Long 1971, p. 124. Voir aussi Clément d'Alexandrie, Stromates, II, 4 ; Cicéron, De natura deorum, I, 43 ; Diogène Laërce, X, 32 : les préconceptions sont la catégorie dont toutes les autres sont dérivées.

12. Ce terme apparait en Hrdt. 35, 45, 46 et 68.

13. De fait, en dehors de l'œuvre de Diogène Laërce, les préconceptions ne sont jamais explicitement posées comme des critères de vérité dans les textes épicuriens qui nous restent.

14. Cf. Sedley 1973. 
Épicure y affirme explicitement qu'il n'y a pas de préconception du temps; si elle ne l'est pas, il se contente de nier que le temps soit lié à un substrat, thèse beaucoup plus faible. Si David Glidden ${ }^{15}$, partisan de l'hypothèse d'une addition postérieure destinée à répondre aux critiques adressées à la théorie des propriétés et accidents, juge lui aussi abusif de voir en $\mathrm{Hrdt}$. 38 une allusion aux préconceptions, l'incertitude persistante concernant la datation des textes d'Épicure, le revirement ultérieur de David Sedley et la correspondance fidèle du De rerum natura avec la Lettre à Hérodote conduisent à conclure que la mention des préconceptions en $H r d t .72$ fait partie du texte original d'Épicure et que Hrdt. 38 évoque également déjà les préconceptions. Le temps fait donc exception à la première règle, car il n'y en a pas de préconception :

En outre, il faut méditer avec force le point suivant : il n'y a certainement pas à mener la recherche sur le temps comme sur le reste, c'est-à-dire tout ce que nous cherchons en un substrat, et que nous rapportons aux prénotions considérées en nous-mêmes [...]. (Hrdt.72.)

Certains, comme Pierre-Marie Morel (nous y avons fait allusion en introduction) ont voulu distinguer la préconception de la « notion première $^{16} \gg$ : la notion première exigée par Épicure dans la formulation de la première règle serait plus large que la simple préconception, qui repose sur la mémoire, ce qui rend possible l'association du temps, sinon à une préconception, du moins à une « notion première ». Une telle hypothèse ne fait cependant que repousser le problème de savoir ce que recouvre le mot temps: qu'il s'agisse d'une préconception ou d'une notion première, qu'associons-nous exactement au temps? C'est ce qu'il est difficile de saisir à la lecture de $\mathrm{Hrdt}$. 72-73, et qu'il est pourtant nécessaire de comprendre, pour garantir la légitimité de cette notion. Pour répondre à cette question, il convient au préalable d'étayer le constat littéral de l'absence de préconception du temps par les raisons conceptuelles susceptibles d'expliquer cette exceptionnalité du temps.

15. Glidden 1985.

16. « Il me semble bien plus éclairant d'admettre que la notion de prôton ennoèma a une extension plus large que celle de prolepse et qu'elle recouvre non seulement des évidences proleptiques, mais aussi des évidences non proleptiques, comme celle qui accompagne l'usage du mot "temps". Dès lors, le fait qu'il n'y ait pas de prolepse du temps, parce que celui-ci ne saurait être abstrait de ce qui prend du temps, ne signifie pas que nous ne puissions en avoir une notion première, en l'occurrence une perception immédiate, une epibolè, et que nous ne sachions pas à quoi le mot "temps" renvoie. » (Morel 2002, p. 210.) 


\section{Les raisons de l'impossibilité d'une préconception du temps}

La préconception ( $\pi p \dot{\lambda} \lambda \eta \psi(\varsigma)$ épicurienne est ainsi définie par Diogène Laërce :

Quant à la prénotion, ils disent qu'elle est comme une perception, ou une opinion droite, ou une notion, ou une conception générale que nous avons en réserve en nous, c'est-à-dire la mémoire de ce qui nous est souvent apparu en provenance du dehors, par exemple quand on dit que « telle sorte de chose est un homme ». En effet, en même temps que l'on prononce « homme », aussitôt par la prénotion on pense à une image de l'homme, du fait que les sensations précèdent. Et donc pour tout nom, ce qui en premier est mis à ses côtés est clair. Et nous n'aurions pas entrepris de chercher ce que nous recherchons, si nous ne l'avions pas connu auparavant, comme lorsqu'on dit : « Ce qui se trouve là-bas est un cheval ou un bœuf »; car il faut par la prénotion avoir connu un jour la forme du cheval et du bœuf. Et nous n'aurions pas non plus donné un nom à quelque chose si auparavant nous n'avions pas connu son image par la prénotion. Les prénotions sont donc claires. (Diogène Laërce, $\mathrm{X}, 33$.)

Il faut distinguer l'aspect génétique de la préconception de son aspect épistémologique. La genèse de la préconception s'effectue à partir des sensations, la répétition de sensations semblables dans notre âme finissant par imprimer une notion générale. Une fois formée, la préconception devient un critère de vérité, car elle permet une anticipation de la perception, c'est-à-dire la reconnaissance et l'identification d'un type de choses (《c'est un homme»), ou d'une chose singulière («c'est Socrate $\gg)$. Les deux caractéristiques essentielles de la préconception sont ainsi le fait qu'elle est issue de la répétition d'un même type d'expérience perceptive, puis sa cristallisation sous la forme d'un contenu de pensée, auquel correspond un mot. Ce second aspect est explicitement nié par Épicure, lorsqu'il précise en $H r d t$. 72 que le temps ne doit pas être rapporté « aux prénotions considérées en nous-mêmes ». Dans un article relativement récent consacré à la question du temps épicurien ${ }^{17}$, Francesco Verde suggère que cette précision signifie que la préconception du temps dépend toujours de réalités extérieures ${ }^{18}$; cependant, comme il le remarque lui-même immédiatement après, toute préconception vient de l'extérieur, et l'on ne peut donc pas en tirer la conclusion qu'il existerait deux types de préconceptions, l'une toujours liée à la perception de

17. Cf. Verde 2008.

18. Comme l'indique chez Lucrèce, à propos de la perception du temps, l'expression ab ipsis rebus (nous percevons le temps « à partir des choses mêmes 》) : tempus item per se non est, sed rebus ab ipsis consequitur sensus... (De rerum natura, I, 459-460). 
réalités extérieures, comme le serait celle du temps, l'autre pouvant faire l'objet d'une cristallisation interne. Accident d'accidents, le temps est toujours évalué par rapport à des accidents eux-mêmes connus dans des corps, ce qui permet de fixer une préconception de ces accidents. C'est ce qui n'est pas possible pour le temps, à cause de sa nature même ${ }^{19}$; il convient à présent de préciser pourquoi.

\section{L'argument de circularité}

Le rejet d'une préconception du temps pourrait sembler tout simplement reposer sur une objection de circularité, dans la mesure où le concept même de préconception fait appel au temps, par la répétition qui constitue, à l'origine, la préconception ou encore par celle qui la constitue perpétuellement, à chacune de ses occurrences, si l'on suit l'analyse de David Glidden et sa critique de l'interprétation psychologique communément faite de la préconception épicurienne comme pure représentation cognitive et contenu mental. En s'appuyant sur l'étude d'un passage des Esquisses pyrrhoniennes ${ }^{20}$ dans lequel Sextus Empiricus défend la thèse épicurienne de l'inutilité de la définition contre la position stoïcienne inverse, Glidden met en effet en lumière l'aspect dynamique de la préconception épicurienne. Sextus Empiricus oppose dans ce passage deux conceptions de la «notion première ». La première, stoïcienne, est d'ordre psychologique : la notion première, que la définition a pour vocation de rassembler en une formule, y est purement représentative, permettant l'anticipation de l'expérience. La seconde, épicurienne, n'est pas représentative, mais recognitive, c'est-à-dire qu'elle est indissociable d'une opération mentale de reconnaissance associant un mot à un état de choses. Les préconceptions ou «notions premières » épicuriennes (le terme technique de préconception étant, selon David Glidden, postérieur à la Lettre à Hérodote) sont ainsi selon lui des représentations temporaires, transitoires, susceptibles de se modifier au fur et à mesure de l'accumulation des expériences. Elles sont proches des symptômes qui s'accumulent dans la mémoire, selon la médecine empiriste (dont on connaît aujourd'hui les

19. Cf. Glidden 1973, p. 210-211: « [...] il ne peut pas y avoir de préconception du temps, car le temps comme tel représente une création cognitive, par opposition à un état persistant dont on peut faire l'expérience régulière dans la nature » (" $[\ldots]$ there cannot be a prolepsis of time, because time as such represent a cognitive creation as opposed to a persistent condition regularly experienced in nature"). Épicure n'exclut pas cependant, selon lui, la possibilité d'une préconception de « régularité de régularités », à distinguer du concept de temps, et qu'il caractérise comme pouvant correspondre à une sorte de structure persistant à travers le passage du jour à la nuit, d'une affection à une autre ou à l'absence d'affection. Le contenu de cette notion est cependant, nous semble-t-il, obscur.

20. Cf. Sextus Empiricus, HP II, 212. 
liens étroits avec l'épicurisme), qui décrit la généralisation progressive des observations du médecin comme une reconnaissance qui s'effectue spontanément, sans raisonnement ni interprétation. La préconception ne consiste pas en une représentation cognitive autonome, mais en un acte, constamment répété, de reconnaissance de divers états du monde comme similaires. L'argumentation de Glidden a pour intérêt de rappeler que la préconception, même conçue comme un contenu cognitif, est indissociable d'un acte dynamique de répétition sensorielle, et que, loin d'être statique, elle est nécessairement revivifiée en permanence par sa propre répétition.

Or, dès lors qu'une préconception consiste en un processus qui s'inscrit dans le temps, n'est-il pas circulaire d'envisager une préconception du temps ? Comment passer de la répétition d'une perception à la perception d'une répétition ? Cela ne suppose-t-il pas d'avoir déjà une préconception du temps ? Et, dans ce cas, comment parvenir à la préconception du temps sans déjà en disposer ? La constitution de la préconception du temps ne requiert pas, cependant, de disposer déjà de cette préconception, car si le temps est l'une des conditions d'acquisition des préconceptions, il n'est pas pour autant intégré au contenu cognitif de cellesci. Par conséquent, le fait que la mémoire et l'anticipation présupposent un processus temporel n'interdit pas de concevoir une mémoire et une anticipation de l'expérience du temps, c'est-à-dire de la temporalité de l'expérience. Aucune circularité n'est donc à craindre et ce sont d'autres raisons qui s'opposent à l'existence d'une préconception du temps.

« Le temps », n'étant pas quelque chose, ne peut faire l'objet ni d'une perception, ni d'une déduction

Au livre VII d'Adversus mathematicos, Sextus affirme que la préconception permet d'établir un lien entre le oquaivov (le « signifiant », ou le mot) et la chose, désignée en l'occurrence par le terme $\tau \nu \gamma \chi \dot{\alpha} v o v$, littéralement « ce qui arrive », ou encore « ce qui se trouve être là ». Ce dernier terme est particulièrement pertinent dans le cadre de la physique épicurienne, où atomes et composés atomiques sont en évolution permanente, au sens propre du mot, puisqu'ils sont toujours en mouvement: une « chose » n'est jamais, en effet, que l'état provisoire d'une configuration atomique - ce qui n'empêche pas certaines configurations de se répéter, permettant ainsi le processus de reconnaissance et de désignation, ni d'être stables pendant une durée conséquente. Il s'agit bien, cependant, d'un objet identifiable par la perception, la préconception impliquant l'anticipation d'une expérience répétable, et c'est précisément cela qui semble constituer un obstacle de principe à la formation d'une préconception du temps. En effet, lorsque nous appréhendons une durée, nous 
ne percevons pas quelque chose, mais seulement l'accident temporaire ${ }^{21}$ d'un accident, si bien que lorsque nous parlons $d u$ temps, nous ne faisons référence à rien d'autre qu'à divers accidents qui durent plus ou moins longtemps. Il ne peut donc y avoir de préconception du temps pour luimême, puisqu'il est toujours perçu relativement à d'autres réalités, dont il constitue en quelque sorte un aspect. Qu'il soit permanent ou provisoire, un accident n'est certes lui-même qu'un agrégat considéré sous un angle particulier (par exemple sa couleur), mais cela ne l'empêche pas de faire l'objet d'une préconception, qui peut ensuite s'appliquer à divers corps. Il existe bien des préconceptions correspondant à des abstractions, comme par exemple celle de la justice : nous pouvons énoncer les caractéristiques et anticiper l'appréhension d'une action juste ${ }^{22}$. Toutefois, l'appréhension de la temporalité des choses ne peut en tant que telle faire l'objet d'une anticipation, car, précisément, ce n'est pas la temporalité que nous percevons, mais tel ou tel accident, perçu sous l'angle de sa durée. Rien, dans cette expérience, ne paraît pouvoir faire l'objet d'une mémorisation sous la forme d'un contenu cognitif. On pourrait tout au plus concevoir qu'il existe des préconceptions correspondant à des durées précises, que l'on reconnaîtrait pour les avoir déjà éprouvées ; ce serait cependant une préconception paradoxale, puisqu'elle ne pourrait intervenir que rétrospectivement (une fois la durée écoulée), si bien que l'anticipation n'informerait pas directement l'expérience du sujet, mais sa réflexion rétrospective sur l'accident qui viendrait d'avoir lieu. En outre, il y aurait alors autant de préconceptions que de durées, ce qui contredirait l'unicité nécessaire à une préconception $d u$ temps. Le contenu d'une préconception du temps semble donc impossible à préciser.

S'il ne fait pas l'objet d'une induction, le temps ne pourrait-il pas faire l'objet d'une déduction, comme c'est le cas pour l'existence des atomes? Épicure exclut catégoriquement une telle possibilité : «Celui-ci en effet ne requiert pas une démonstration [...] » (Hrdt. 73). Le temps est associé à une forme d'évidence (il faut « nous référer à l'évidence même, selon laquelle nous parlons de "temps long" ou de "temps court" »), il informe

21. Un $\sigma \dot{\nu} \mu \pi \tau \omega \mu \alpha$, et non un $\sigma u \mu \beta \varepsilon \beta \eta \kappa o ́ s$ qui accompagne le corps pendant toute l'existence de celui-ci.

22. Les épicuriens ont une conception conventionnaliste de la justice : il y a bien une préconception du juste, mais celle-ci ne relève pas d'une vertu de justice ; la justice repose cependant sur la notion d'utilité à la communauté, ce qui explique l'existence d'une préconception de la justice, fondée sur ce principe universel d'utilité commune, malgré les variations ponctuelles en fonction des lieux et des circonstances (voir Maximes capitales, XXXIII-XXXVIII). On peut encore citer la préconception de la causalité, évoquée par

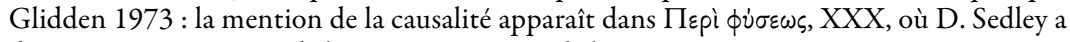
découvert une mention de la « préconception de la cause ». 
naturellement notre expérience, et il ne fait donc pas partie des réalités inévidentes ou invisibles. Il nous faut donc conclure qu'il n'y a pas de préconception du temps, ni induite, ni déduite, et que l'appréhension des durées ne donne pas lieu à la formation d'une préconception. Le paradoxe initial reste dès lors entier : la notion et le mot temps sont légitimes, sans toutefois faire l'objet d'une préconception. Quel est le point commun de nos différentes expériences de la durée, à l'origine de l'évocation $d u$ temps ? Que recouvre ce vocable ? C'est ce qu'il nous faut élucider pour achever notre analyse de $\mathrm{Hrdt}$. 72-73.

\section{La substitution de l'analogie à la préconception}

La première étape de la partie positive de la méthode recommandée par Épicure pour une enquête sur le temps est une analogie, qui doit venir remplacer le recours à la préconception :

[...] nous devons, par analogie, nous référer à l'évidence même, selon laquelle nous parlons de «temps long » ou de «temps court », d'une manière conforme <à cette évidence>. Et il ne faut pas changer les termes pour d'autres qui seraient meilleurs, mais il faut se servir à son propos de ceux qui existent; et il ne faut pas non plus lui attribuer quelque autre chose, dans l'idée que son être est identique à cette caractéristique, mais il faut surtout soumettre à épilogisme cette seule chose : à quoi nous lions ce caractère qui lui est propre, et par quoi nous le mesurons. Celui-ci en effet ne requiert pas une démonstration mais un épilogisme, à savoir que nous le lions aux jours et aux nuits et à leurs parties, tout comme aux affections et aux non-affections, aux mouvements et aux repos, concevant en retour par rapport à ces réalités un certain accident particulier, en référence auquel nous prononçons le mot « temps ». (Hrdt. 72-73, trad. Balaudé modifiée.)

\section{Les deux sens de l'analogie dans la méthode épicurienne}

L'analogie est, avec l'épilogisme et la déduction, la principale forme de raisonnement utilisée par Épicure. Elle permet à la fois de former des idées générales et d'avoir accès aux réalités inévidentes :

De là vient que les réalités inévidentes sont à rendre manifestes à partir de ce qui apparaît; de fait, toutes les pensées tirent leur origine des sensations, aussi bien par la rencontre, l'analogie, la ressemblance, la composition, ce à quoi le raisonnement apporte aussi sa contribution. (Diogène Laërce, X, 32, trad. Balaudé modifiée.)

Nous choisissons de modifier la traduction de Jean-François Balaudé, considérant que par દ̇ $\pi \iota v o i \alpha \iota$ sont évoqués tous les types de pensées, y com- 
pris celles liées à la perception (nous reprenons ici l'analyse proposée par Julie Giovacchini dans sa thèse $\left.{ }^{23}\right)$. Lorsque la rencontre $(\pi \varepsilon p i \pi \tau \omega \sigma \iota \varsigma)$ ne suffit pas à produire directement une préconception, l'analogie, la ressemblance et la composition interviennent pour pallier la défaillance de la perception. Ce qu'Épicure appelle analogie recouvre en vérité une procédure assez vague, qui ne correspond pas seulement au sens moderne de la répétition d'un rapport permettant, à partir de la connaissance de trois termes, de déduire le quatrième. Épicure utilise ce terme en un sens non technique, qui consiste à mettre en évidence des ressemblances entre des réalités perceptibles, ou entre des réalités perceptibles et d'autres qui ne le sont $\operatorname{pas}^{24}$ : dans le premier cas, il s'agit simplement de mettre en lumière des similarités; dans le second, l'analogie permet de former une préconception de réalités inévidentes - par exemple les atomes.

Le premier type d'analogie apparaît par exemple en Men.127, où la classification des désirs est présentée comme le résultat d'un raisonnement analogique ${ }^{25}$ consistant simplement, semble-t-il ${ }^{26}$, à comparer entre eux les désirs :

Et il faut voir, en raisonnant par analogie, que parmi les désirs, certains sont naturels, d'autres vides, et que, parmi les désirs naturels, certains sont nécessaires, d'autres seulement naturels; et parmi les désirs nécessaires, certains sont nécessaires au bonheur, d'autres à l'absence de perturbations du corps, d'autres à la vie même. (Men. 127.)

Un exemple du second type d'analogie est l'existence d'une grandeur minimale dans l'atome :

Enfin, il faut bien penser que ce qu'il y a de plus petit dans la sensation n'est pas semblable à ce qui peut être parcouru, et n'en est pas non plus

23. Cf. J. Giovacchini, La Méthode épicurienne et son modèle médical, thèse de doctorat soutenue en mars 2007 à l'université Paris X-Nanterre, p. 162. Nous remercions l'auteur de nous avoir permis de consulter son travail.

24. Cf. E. Asmis : «Ce verbe composé, tout comme les formes analogia et analogos, est utilisé dans le sens d'un recensement des ressemblances, qu'il s'agisse de ressemblances entre les phénomènes ou de ressemblances entre les phénomènes et les choses non observées. 》 ("This compound [analogizein], together with the forms analogia and analogos, is used in the sense of calculating similarities, whether they are similarities among the phenomena or similarities between the phenomena and things that are unobserved." Asmis 1984, p. 177.)

25. Le terme utilisé est ả $\nu \alpha \lambda \circ \gamma\llcorner\sigma \tau \dot{\varepsilon} \circ \nu$, terme utilisé également dans le passage sur le temps.

26. J.-F. Balaudé note le caractère vague de l'analogie dans ce passage: «c'est un raisonnement analogique, fondé probablement (car cela reste quelque peu obscur) sur la saisie des similitudes entre les divers types de plaisirs dont nous faisons l'expérience. » (Balaudé 1999, Introduction au livre X, p. 1214.) 
totalement dissemblable, de telle sorte qu'il présente un caractère commun avec ce qui se laisse parcourir, bien qu'on ne distingue pas en lui de parties. Mais quand, en raison de la ressemblance que procure ce caractère commun, nous pensons distinguer quelque chose de lui, à savoir une partie antérieure, et une partie postérieure, nous parvenons nécessairement à l'égalité entre elles. [...] Il faut considérer que cette analogie vaut pour l'élément le plus petit dans l'atome. En effet, il est évident que celuici differe par la petitesse de ce qui est observé dans la sensation mais la même analogie vaut ; car précisément, que l'atome est pourvu d'une grandeur, c'est ce que nous avons affirmé, en suivant cette analogie sensible, nous contentant d'agrandir quelque chose qui est petit. (Hrdt. 58-59.)

L'analogie entre les corps que la perception peut parcourir et leurs extrémités (la plus petite partie d'un corps que nous puissions percevoir), puis entre ces extrémités et les atomes, fonde la déduction de l'existence de parties minimales à l'intérieur même de l'atome. Elle a pour justification physique la commensurabilité des corps et des atomes entre eux, en vertu de l'atomisme de la matière et de la grandeur ; elle repose également sur l'analogie du non-sensible au sensible. Il s'agit ici d'une analogie au sens strict, puisque l'existence du minimum de grandeur dans l'atome est déduite comme devant avoir le même rapport à l'atome que la plus petite longueur perceptible à l'objet auquel elle appartient.

Loin d'être incompatible avec la préconception, l'analogie peut donc, selon les deux modalités que nous venons d'évoquer, contribuer au raisonnement par lequel on obtient cette dernière. Aucun de ces deux usages de l'analogie n'est cependant applicable au cas du temps, et c'est pourquoi il doit s'agir ici d'un substitut, et non d'un préalable, à l'obtention d'une préconception.

\section{Le sens particulier de l'analogie en Hrdt. 72-73}

[...] nous devons, par analogie, nous référer à l'évidence même, selon laquelle nous parlons de « temps long » ou de « temps court », d'une manière conforme <à cette évidence>. ( $H r d t$. 72, trad. Balaudé modifiée.)

L'analogie qui permet d'obtenir la connaissance d'une réalité inévidente à partir d'une réalité évidente - second des deux cas de figure évoqués précédemment - n'est pas en jeu dans le cas du temps, puisque dans ce dernier cas l'analogie vise l'évidence selon laquelle nous parlons d'un temps long ou d'un temps court. Reste donc l'analogie comparative ; celle-ci peut être comprise en deux sens dans le cas du temps, selon qu'on la lie à la méthode d'inférence inductive, ou qu'on la comprend comme une analogie entre les durées elles-mêmes. Selon une première lecture, l'analogie concerne la comparaison entre elles de choses que nous appelons toutes des durées, et de cette comparaison naît l'idée de mesure 
temporelle. Celle-ci repose elle-même sur l'évidence du long et du court temporels, évidence qui s'impose à nous par une autre analogie, avec ce que nous appelons long et court dans l'espace - la mesure des corps et du vide, tous deux principes de la physique, apparaît donc ici comme première par rapport à celle des durées. Selon une seconde lecture, les durées sont analogues les unes aux autres, et cela à strictement parler, en un sens technique, du fait qu'elles sont toutes commensurables entre elles, tout comme les parties minimales des atomes rendent toutes les grandeurs spatiales commensurables.

Considérons à présent plus en détail le premier sens de l'analogie comparative qu'Épicure mentionne à propos du temps. La première question qui se pose concerne la nature de l'évidence à laquelle doit se référer l'analogie : est-ce l'évidence du temps lui-même, celle du long et du court, ou celle des réalités qu'accompagne le temps (qui sont les jours et les nuits, les affections et les absences d'affections, les mouvements et les repos) ? La seconde question concerne le problème méthodologique de compatibilité qu'il semble y avoir entre l'évidence, l'absence de préconception, et la nécessité de recourir à une analogie.

\section{À quelle évidence se réfère l'analogie?}

L'évidence en jeu pourrait être celle d'une perception directe du temps - comme le suppose Carlo Giussani ${ }^{27}$, suivi par d'autres interprètes tels que Cyril Bailey $^{28}$, Anke Manuwald ${ }^{29}$ et Fritz Jür $\beta^{30}$ - ; mais, dans ce cas, l'analogie n'aurait pas lieu d'être, et nous disposerions d'une préconception du temps (fondée directement sur la sensation) $)^{31}$. Une deuxième hypothèse est que l'évidence qui sert de point de départ à l'analogie est tout simplement celle du long et du court, dont nous faisons d'abord l'expérience en mesurant l'espace. L'évidence serait alors celle de la mesure, prioritairement spatiale, mais que nous appliquons, par analogie, au

27. Cf. Giussani 1896-1898; dans son commentaire de De rerum natura I, 30-31, Giussani comprend $\dot{\varepsilon} v \dot{\alpha} p \gamma \eta \mu \alpha$ comme se référant à «l'intuition du temps ».

28. Cf. Bailey 1926, p. 241-242.

29. Cf. Manuwald 1972, p. 76-78.

30. Cf. Jürß 1977.

31. C'est la position de F. Jürß, qui pense qu'il s'agit d'une préconception exceptionnelle : « La préconception du temps est donc une exception, car on ne peut la voir en nous par les yeux de l'esprit, comme c'est le cas pour les autres formes de pensée conceptuelles, mais elle se constitue d'abord par abstraction à partir des symptômes, c'est-àdire de préconceptions de première instance [...]. » ("Dennoch ist die Prolepse der Zeit ein Sonderfall, weil mann sie nicht wie den anderen begrifflichen Gedankenformen mit den geistigen Auge in uns selbst erschauen kann, sondern weil sie sich erst in Abstraktion von den Symptomen bzw. Prolepsen erster Instanz konstituiert [...]”. Jürß 1991, p. 108.) 
temps. Une troisième possibilité est que l'évidence à partir de laquelle se fait l'analogie soit celle des réalités dont le temps est l'accident et que nous disons être plus ou moins longues. C'est l'avis d'Elizabeth Asmis ${ }^{32}$, ainsi que d'Anthony Long et David Sedley ${ }^{33}$ : ce qui est évident, selon eux, c'est ce que nous percevons directement et identifions grâce aux préconceptions selon la méthode habituelle, à savoir les phénomènes auxquels nous associons le mot temps, qui sont donc le point de départ de l'analogie. Nous ne connaissons le mot temps que dans la mesure où nous savons ce que nous voulons dire par «durée d'une maladie » ou «longueur du jour », sans jamais avoir de connaissance du temps isolément des durées, ni donc des accidents que celles-ci qualifient.

Cette réponse nous semble la plus satisfaisante, car elle est cohérente avec l'allusion faite dans la suite du texte à l'épilogisme portant sur les réalités qu'accompagne le temps («Celui-ci en effet ne requiert pas une démonstration mais un épilogisme, à savoir que nous le lions aux jours et aux nuits et à leurs parties, tout comme aux affections et aux nonaffections, aux mouvements et aux repos », $H r d t$. 73) : nul besoin d'une démonstration, car la perception du temps se fait pour ainsi dire « à même » l'évidence de la perception des accidents. L'analogie qui doit se référer à l'évidence des réalités pourvues d'une durée doit cependant s'effectuer aussi entre le long et le court, mesurés dans ces réalités ellesmêmes : on rejoint ici la deuxième hypothèse précédemment citée. L'analogie consiste nécessairement, en effet, en une comparaison entre les durées des accidents que nous percevons : nous rapportons une mesure temporelle (toujours immanente à un accident particulier) à d'autres qui l'ont précédée, et nous nous forgeons ainsi une idée de la durée habituelle de certains types de réalités (la durée d'une journée, par exemple). La question de savoir s'il y a lieu de parler d'une commensurabilité réelle des durées entre elles rejoint celle d'un éventuel atomisme du temps épicurien, qui mérite un traitement séparé dépassant les limites de cet article.

En l'absence de préconception du temps, comme de notion générale de ce qu'ont en commun toutes les durées, l'analogie mobilisée dans le cas $\mathrm{du}$ temps est tout à fait singulière, puisqu'elle prend la forme d'un processus perpétuellement ouvert, qui n'aboutit jamais à aucune abstraction. Épicure insiste à cet égard sur le fait que le temps se manifeste toujours de façon irréductiblement particulière, sous la forme de telle ou telle durée : « concevant en retour par rapport à ces réalités un certain

32. Cf. Asmis 1984, p. 33, n. 35 : « The evident occurrence, as I interpret it, is a property that is perceived to last for a short or long time. Epicurus sums up these properties as nights and days, motions and rests, and feelings or absences of feeling. »

33. Cf. Long \& Sedley 2001, I, p. 85. 
accident particulier ( $` \delta ı ่ \nu \tau \iota \sigma \dot{\nu} \mu \pi \tau \omega \mu \alpha$ ) en référence auquel nous prononçons le mot “temps” » (Hrdt. 73). Cet accident $(\sigma \dot{\nu} \mu \pi \tau \omega \mu \alpha)$ n'est pas une propriété ( $\sigma u \beta \varepsilon \beta \eta \kappa o ́ s)$, et il ne saurait l'être, contrairement aux autres qualités, qui peuvent indifféremment être des accidents (provisoires) ou des propriétés (« définitives », c'est-à-dire durant aussi longtemps que l'agrégat qu'elles qualifient) : il est en effet, pour reprendre la formule de Démétrius Lacon, un « accident d'accidents ${ }^{34}$ ». Le temps ne peut jamais être qu'un accident particulier, par opposition à la généralité de la préconception, car il est par nature labile et éphémère.

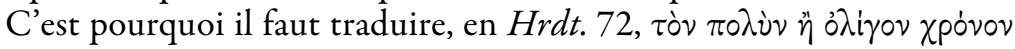

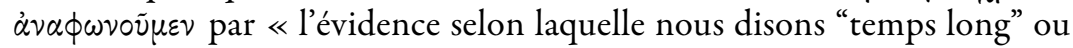
"temps court" ${ }^{35}$ et non, comme il serait grammaticalement possible de le faire, «selon laquelle nous parlons $d u$ temps comme long ou court » (ou «selon laquelle nous disons que le temps est long ou court »). Une telle traduction a en effet le mérite de rendre manifeste que le temps n'existe pas sous la forme d'une continuité dans laquelle seraient les choses, qu'il n'existe pas indépendamment des durées. Le temps épicurien n'est pas une réalité continue constituant en quelque sorte un médium sous-jacent, dont les différentes durées perçues seraient autant de portions. Un temps long, c'est toujours un temps long en comparaison d'un temps moins long. Pour Épicure, le mot temps ne renvoie ainsi à rien d'autre qu'à une perpétuelle comparaison entre durées, qui s'effectue de manière immanente à notre perception des accidents, c'est-à-dire des durées particulières; il est donc de nature intrinsèquement comparative. Parler de temps, c'est alors toujours seulement exprimer la mise en rapport d'une durée avec une autre. La comparaison porte en elle-même une évidence : ce jour est par exemple plus long que la nuit qui l'a précédé, et ce rapport quantitatif nous apparaît de façon manifeste; en revanche, en percevant la durée de ce jour ou de cette nuit, nous ne faisons pas l'expérience de la temporalité ou de la durée en général, et c'est en ce sens que nous n'avons pas de préconception du temps. Épicure rejette ainsi toute idée du temps comme entité séparée, et a fortiori comme cadre fixe servant d'environnement à toutes choses. Dans un article sur la conception aristotélicienne du temps, J.-F. Balaudé ${ }^{36}$ insiste sur l'ambiguïté de l'« être dans le temps » aristotélicien. En particulier, il repère

34. $C f$. n. 9 ci-dessus.

35. C'est le parti également pris par Conche 1977, p. 177 («il faut prendre en compte l'évidence même suivant laquelle nous parlons d'un temps “long" ou "court" ») et par Balaudé 1999, p. 1283 (« nous devons, par analogie, nous référer à l'évidence même, suivant laquelle nous parlons d'un temps long ou court »).

36. Voir Balaudé 2005, p. 154 sq., en particulier p. 161-163. 
et commente la tension suivant laquelle Aristote tente à la fois de reconduire tout mouvement à un certain temps («être dans un temps »), et de garantir l'unité de tous les temps par leur insertion dans un seul et même temps naturel (《être dans le temps »). À cet égard, Épicure radicalise la désontologisation aristotélicienne du temps : non seulement « être dans le temps », mais même «être dans un temps » (au sens d'avoir une certaine durée) devient une expression trop abstraite pour ne pas induire en erreur.

La contradiction apparente entre l'évidence du temps, son absence de préconception, et le recours à l'analogie

On comprend ainsi mieux comment résoudre le second problème qui se pose à propos de la procédure analogique concernant le temps : le temps n'est en effet ni directement évident (puisqu'il faut faire une analogie), ni une réalité inévidente (puisque lui est associée une forme d'évidence). Cette singularité s'explique par le caractère très particulier de notre appréhension du temps : celle-ci consiste, non pas en la reconnaissance d'un contenu notionnel, mais en une analogie ouverte et sans cesse recommencée entre les différentes durées, que nous percevons toujours comme relatives les unes aux autres. La perception du temps en reste ainsi toujours à un niveau horizontal, s'épuisant dans la comparaison des durées les unes aux autres, sans qu'aucune notion abstraite de durée ou de généralité puisse être extraite de ce processus de comparaison, et ainsi y mettre un terme. L'appréhension du temps relève d'un processus qui met en relation diverses réalités évidentes, et ce n'est qu'en rapport à un accident survenant à même ce processus qu'est utilisé le mot temps : nous concevons « en retour par rapport à ces réalités un certain accident particulier, en référence auquel nous prononçons le mot “temps" » ( Hrdt. 73). Le mot temps renvoie donc à une modalité de la mémoire que nous avons d'une série de sensations, et ainsi à un accident cognitif de notre perception.

On comprend alors quel sens particulier prend, dans le cas du temps, l'opposition épicurienne du langage ordinaire et du langage savant : faire correspondre au mot temps une préconception, c'est déjà dénaturer le temps en une entité séparée, mais aussi et d'abord dénaturer les expressions temporelles en des expressions savantes, comme s'il y avait quelque chose d'abstrait à comprendre sous ces mots. Faire usage du langage ordinaire en parlant $d u$ temps fait donc paradoxalement déjà courir le risque de tomber dans le langage savant, car, dans le cas du temps, le langage ne doit pas, comme d'ordinaire, refléter une sensation (ou désigner le résultat d'une démonstration), mais codifier une activité de comparaison qui prend place de part en part dans le domaine de l'évident 
(puisqu'il s'agit de rapporter de façon évidente l'une à l'autre des durées elles-mêmes observées de manière évidente, à même des réalités évidentes). Ainsi le régime ordinaire, directement représentatif, du langage, tend-il à devenir, dans le cas du temps, un régime savant, qui suggère une préconception supposée, mais en réalité absente. Le risque de confusion n'en est que plus grand, de même, par suite, que la nécessité de délimiter le bon usage de nos mots. À la limite, quiconque veut faire un usage correct du langage devrait éviter de parler du temps en l'hypostasiant, et il ne faut pas supposer de signification à un discours prétendant porter sur « le temps $\gg$. Le langage ordinaire doit se déprendre de son fonctionnement habituel, pour respecter les conditions d'émergence de nos mentions du temps, et les refléter fidèlement : c'est tout le sens de $\mathrm{Hrdt}$. 72-73.

Épicure ne se contente pas de substituer l'analogie à la préconception ; il réfute également la démonstration au profit de l'épilogisme, qu'il fait porter sur trois couples d'accidents. Il nous reste à présent à analyser le fonctionnement de cet épilogisme, qui vient compléter le recours à l'analogie, pour achever notre élucidation du processus cognitif tout à fait original qui accompagne la perception du temps.

\section{La collaboration de l'épilogisme et de l'analogie dans l'appréhension du temps}

Celui-ci en effet ne requiert pas une démonstration mais un épilogisme, à savoir que nous le lions aux jours et aux nuits et à leurs parties, tout comme aux affections et aux non-affections, aux mouvements et aux repos, concevant en retour par rapport à ces réalités un certain accident particulier, en référence auquel nous prononçons le mot «temps ». (Hrdt. 73, trad. Balaudé modifiée.)

Si l'épilogisme n'a pas de sens explicite dans le corpus épicurien, le texte de $H r d t .72$ est l'un des rares passages où cette notion joue un rôle central. L'épilogisme peut, de manière générale, être défini comme une association de perceptions plus ou moins consciente ${ }^{37}$, fondée sur une observation durable et permettant d'associer plusieurs phénomènes ${ }^{38}$. La

37. Il existe à ce propos une polémique entre Philip De Lacy et Graziano Arrighetti : pour le premier, l'épilogisme est un raisonnement, alors que pour le second, l'épilogisme relève d'une activité naturelle de l'esprit; comme nous allons l'expliquer, il nous semble que, dans le cas du temps, l'épilogisme correspond à une façon de percevoir selon la durée qui s'effectue naturellement, tout en étant couplé à une analogie qui, elle, est bien un raisonnement. Cf. P.H. \& E.A. De Lacy 1941 ( $\left.{ }^{2} 1978\right)$, et Arrighetti 1961 ( $\left.{ }^{2} 1973\right)$.

38. Dans le De signis, Philodème affirme que l'épilogisme prépare l'analogie, en mettant en évidence les ressemblances et différences entre les phénomènes, et notamment 
différence avec l'analogie entre phénomènes évidents est la suivante: l'épilogisme repose sur une accumulation continue d'observations, et non sur une simple mise en rapport, et il ne met en jeu que des phénomènes observables.

On peut ainsi comprendre que Hrdt. 72 fasse du temps l'objet par excellence d'un épilogisme: l'impression de durée que nous ressentons lors du déroulement d'un phénomène (d'un mouvement, par exemple) accompagne en effet par principe ce phénomène tout le long de son déroulement, sans rien y ajouter, si ce n'est justement la mise en rapport des différentes étapes de ce déroulement au titre de moments successifs d'un même phénomène. La perception de la durée d'un accident n'est autre, en effet, que l'observation des différentes phases d'un phénomène y compris son apparition et sa disparition, si bien que chaque perception de durée est de nature épilogistique.

L'examen du début du passage nous a conduit à supposer que le terme d'analogie est utilisé en un sens très particulier, puisqu'il s'agit d'une analogie qui n'aboutit pas à un résultat abstrait. De son côté, l'épilogisme est manifestement ici à comprendre comme la simple observation de ce à quoi nous lions notre vocabulaire temporel : les jours, les nuits et leurs parties; les affections et non-affections; ainsi que les mouvements et les repos. L'épilogisme a pour rôle l'observation des choses liées au mot temps, ce qui est cohérent avec la réfutation de l'existence d'une préconception du temps, puisque c'est toujours aux réalités évidentes auxquelles est associé le temps qu'il faut revenir pour penser ce dernier. Ce qui est sous-jacent au mot temps ne peut être obtenu que par une réflexion rétrospective fondée sur l'observation de la diversité des réalités auxquelles est associé le temps et sur la compréhension de l'attribution comparative, à chacune d'entre elles, d'une certaine durée (toujours relativement à une autre prise comme repère temporaire) : notre perception du temps prend toujours la forme d'une comparaison entre une durée perçue présentement et une durée précédente. L'épilogisme désigne donc ici l'opération mentale suivant laquelle nous enregistrons le déroulement de différents phénomènes et les conservons en mémoire sous la forme de durées homogènes les unes aux autres. L'analogie intervient alors pour mettre les durées observées par épilogisme comme longues ou courtes les unes en rapport avec les autres, sans toutefois (c'est là sa particularité) conclure à l'existence d'une propriété commune autre que la capacité de chaque accident qu'accompagne le temps d'être comparable à d'autres

en analysant le caractère éventuellement fortuit des ressemblances, ce qui permet de les prendre ou non pour fondement d'une analogie. 
accidents sous le rapport de la quantité temporelle. Qu'ont en commun toutes les durées singulières ? Précisément, uniquement de pouvoir être rapportées les unes aux autres (dans certaines limites) du point de vue du long et du court. Or cette comparabilité ne constitue aucunement un contenu cognitif fixe (qui serait l'objet de notre perception « du » temps), mais s'épuise en l'effectuation de comparaisons, toujours concrètes et particulières, de durées entre elles : elle ne donne accès à aucune qualité commune.

Il est remarquable à cet égard que les réalités sur lesquelles porte l'épilogisme soient trois couples d'accidents contraires : les jours et les nuits (et leurs parties), les affections et absences d'affections, les mouvements et les repos. La liste dressée par Épicure mentionne uniquement des cas d'alternances. On peut supposer qu'il ne s'agit pas d'une coïncidence, et que cela souligne deux caractéristiques essentielles de la perception du temps. Tout d'abord, la genèse de toute perception temporelle est liée à l'apparition et à la disparition d'un accident : c'est seulement une fois qu'un accident n'est plus présent que nous pouvons rétrospectivement en déterminer la durée. En appréhendant une durée particulière, nous ne percevons pas quelque chose qui aurait une consistance ou une texture propre, mais nous mesurons un accident, rétrospectivement. Plus fondamentalement, l'alternance signifie, nous semble-t-il, la succession de deux durées de longueurs naturellement comparables : celles du jour et de la nuit, du mouvement et du repos, de l'affection et de la non-affection. L'évocation de trois alternances suggère donc fortement que la perception et la mesure du temps s'effectuent toujours par comparaison avec une durée proche, c'est-à-dire du même genre et de la même échelle. La durée d'un jour nous semblera en effet plus ou moins longue relativement à celle de la nuit qui l'a précédée, la durée d'un mouvement par rapport à la durée du mouvement ou du repos précédent, et nous évaluerons celle d'un plaisir en fonction de celle de l'absence de plaisir antérieure. En revanche, il n'est guère adéquat, sauf cas particulier, de rapporter la durée d'une affection avec celle d'une nuit, dans la mesure où la première est en général incomparablement plus courte que la seconde.

Notre hypothèse est qu'Épicure fait ainsi référence à l'existence d'échelles naturelles de durées. Nous expérimentons le temps selon des types de durées qui se dégagent simplement du fait qu'une durée est d'abord naturellement comparable à certaines autres, et non à toutes les autres. De même que le mot temps ne recouvre rien d'autre que la comparabilité de principe d'une durée avec d'autres durées, de même un ordre de grandeur de durée ne signifie-t-il rien d'autre que la plus ou moins grande aisance avec laquelle nous rapportons une durée déterminée à telle ou telle autre. On peut alors supposer que les jours et les nuits et 
leurs parties jouent dans la mesure du temps un rôle privilégié parce qu'il s'agit de durées universellement éprouvées, et parce qu'ils correspondent à une échelle de temps médiane, ce qui en fait des unités de mesure commodes, permettant de rendre presque toutes les durées commensurables entre elles. Cela ne signifie pas, en revanche, que le jour ou la nuit représente une unité de mesure abstraite ancrée dans l'ordre naturel des phénomènes. Au contraire, une durée n'est pas en général comparable à toutes les autres : la dernière conséquence à tirer des exemples d'alternances mis en avant par Épicure est en effet qu'il ne saurait exister d'unité de mesure universelle et absolue des durées. Les échelles de durée sont des ordres de grandeurs observés, approximatifs et relatifs, et nullement des unités de mesure objectives et valables pour toutes les durées; elles sont naturelles seulement au sens où il est aisé de prendre par exemple la durée d'un jour (celle du jour qui précède) comme repère dans la mesure de la durée de certains autres phénomènes du même ordre. Notre perception du temps prend toujours d'abord la forme d'un processus consistant à établir un certain type de rapport quantitatif entre accidents du même genre, dans le cadre d'un registre particulier (celui des mouvements, des affections, ou des parties de la journée, qui représentent les principaux ordres de grandeur de durées), sans jamais se résoudre en la perception d'une réalité qui serait la durée.

\section{Conclusion}

À l'issue de cette étude du passage de la Lettre à Hérodote consacré au temps, la réfutation par Épicure de l'existence d'une préconception du temps nous semble attestée, tant par la lettre du texte de $\mathrm{Hrdt}$. 72-73 que par une nécessité conceptuelle liée à la singularité de la conception épicurienne du temps, dont nous avons tenté de reconstituer le détail. Le mot temps n'est pas solidaire d'un contenu de pensée, mais de deux choses: l'ensemble des durées, toujours attachées à un accident particulier, et un processus cognitif actualisé à chaque perception de durée, et dont l'originalité est due à un mélange d'analogie et d'épilogisme. Dans le cas du temps, l'analogie recommandée ne se solde pas par un effet de généralité, comme c'est habituellement le cas lorsque celle-ci donne lieu à une préconception obtenue par comparaison (celle de désir naturel et nécessaire, par exemple) ou par la mise en rapport d'une réalité évidente avec une autre, inévidente (celle de l'atome ou de la partie minimale de l'atome). Au contraire, elle est sans cesse relancée par l'épilogisme, en même temps que celui-ci la contrôle en la limitant aux choses évidentes; en retour, l'analogie sollicite l'épilogisme, en même temps qu'elle le guide, pour la recherche de rapports entre durées et l'établissement d'unités de mesure partielles et provisoires. On peut donc réellement parler, dans le 
cas très particulier du temps, d'une collaboration de l'épilogisme et de l'analogie, qui n'est pas sans rappeler le jugement réfléchissant kantien : l'épilogisme et l'analogie, selon Épicure, doivent collaborer dynamiquement dans le cas du temps comme le font, chez Kant, l'imagination et l'entendement dans le cas du jugement de goût ${ }^{39}$. De même que le jugement réfléchissant consiste en un libre jeu des deux facultés en l'absence d'un concept prédéterminant, de même l'analogie établit-elle des rapports entre durées sur la base de données fournies par l'épilogisme, selon une activité sans cesse réactualisée, sans jamais donner lieu à aucune unité de mesure absolue, ni à aucune idée abstraite de durée en général.

Le temps n'est inféré, sous la forme de durées, qu'en tant que synthèse directement expérimentée (grâce à l'épilogisme) des différents moments observables d'un certain processus, immédiatement comparée (par une analogie) à d'autres durées précédemment mesurées. Il est ainsi le corrélat et le produit dérivé de séries de sensations. C'est pourquoi la règle enjoignant de se limiter au langage ordinaire (selon lequel nous parlons de « temps long » et de « temps court ») et celle recommandant, pour le temps, le recours à l'analogie et à l'épilogisme sont à comprendre comme complémentaires, et comme formant au fond une seule et même règle de méthode à propos du temps : «le temps » n'est rien d'autre qu'une certaine catégorie de long et de court, qu'une série de mesures comparatives qui demeurent adhérentes aux durées qu'elles mesurent, et par là même aux accidents particuliers sur lesquelles portent celles-ci.

Le temps épicurien est ainsi proche, en un certain sens, d'une forme de la perception : celle de la comparabilité deux à deux des durées de même échelle, c'est-à-dire des phénomènes de même ordre de grandeur selon le long et le court (comme notamment les deux phases d'une alternance). Cette forme ne peut pas faire l'objet d'une préconception, puisqu'elle est le corrélat d'une comparaison sans inférence comparative, qui prend la forme d'une combinaison réfléchissante d'épilogisme et d'analogie. Cela ne signifie pas, bien entendu, que le temps ne ferait pas partie de la nature : les durées sont un accident réel des choses. L'« accident particulier, en référence auquel nous prononçons le mot "temps" » évoqué en Hrdt. 73, désigne ainsi avant tout l'ensemble non clos des durées ; cependant, dans la mesure où celles-ci sont toujours perçues les unes par

39. Cf. Kant, Critique de la faculté de juger, $\$ 35$. Kant y parle, à propos du jugement de goût, de l'« animation réciproque de l'imagination dans sa liberté et de l'entendement dans sa légalité ». La «liberté » représentée par l'épilogisme pourrait être ici celle du déroulement de l'expérience tel qu'on l'observe sans rien lui superposer. La « légalité » représentée par l'analogie serait la recherche systématique de rapports permettant de ramener une diversité de durées à une unité (celle justement d'un ensemble de rapports). 
rapport aux autres, il est aussi indissociablement lié à un certain mode de perception des accidents ${ }^{40}$. Le paradoxe de la réflexion sur le temps est qu'elle donne lieu, dans la Lettre à Hérodote, à une reconstruction complexe, alors même qu'Épicure veut éviter toute spéculation théorique. L'obscurité de Hrdt. 72-73 trouve sans doute là sa source: dans la difficulté qu'il y a à décrire le temps comme le simple corrélat de la comparaison des durées par le sujet, en incitant le lecteur à faire de sa propre perception du temps une reconstitution artificielle, précisément pour mieux en comprendre la naturalité.

40. Le temps peut apparaitre en ce sens comme une sorte d'accident non seulement des durées, mais aussi du processus cognitif en jeu lors de chaque perception temporelle. On trouve à ce propos dans les fragments du PHerc. 1413 une allusion qui semble faire du temps l'«accident d'un certain type de représentation »: «[...] quand vous dites connaître le temps non pensé, le temps comme accident d'une certaine représentation »

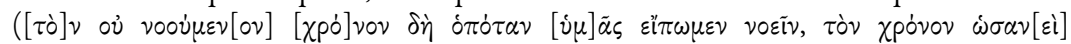
$[\sigma \nu] \mu \beta \varepsilon \beta \eta \kappa o ́ s \tau \iota v ı[\phi \alpha \nu \tau] \alpha \sigma i \alpha[\iota ;$ fr. 37.23 Arrighetti, dans Cantarella \& Arrighetti 1972). L'état du papyrus interdit cependant d'en faire un argument propre à confirmer notre hypothèse. 


\section{BIBLIOGRAPHIE}

AsMIS, E. 1984 : Epicurus' scientific method, Ithaca, 1984 (Cornell studies in classical philology, 42).

ARrigheTTI, G. 1960 : Epicuro , Opere, a cura di -, Turin, 1960 (Classici della filosofia, 4) $\left[{ }^{2} 1973\right.$ (Biblioteca di cultura filosofica, 41)].

BAILEY, C. 1926 : Epicurus, The Extant Remains, with Short Critical Apparatus , Translation and Notes, Londres-Edimbourg-Glasgow, 1926.

BALAUdÉ, J.-F. 1999: Diogène Laërce, Livre X: Épicure, Introduction, traduction et notes, dans M.-O. Goulet-Cazé (éd.), Diogène Laërce, Vies et doctrines des philosophes illustres, Paris, 1999 (La pochothèque. Classiques modernes).

-2005 : « Être dans le temps », dans J.-F. Balaudé et F. Wolff (éd.), Aristote et la pensée du temps = Le Temps philosophique, 11 (2005), p. 145-172.

CANTARella, R. \& G. ARrighetTi 1972 : « Il libro sull tempo (PHerc.1413) dell'opera di Epicuro Sulla natura », Cronache Ercolanesi, 2 (1972), p. 5-46.

CONCHE, M. 1977 : Épicure, Lettres et maximes, Introduction, texte et traduction avec notes, Villers-sur-mer, 1977.

De Lacy, PH. H. \& E. De Lacy 1978 : Philodemus, On methods of inference, edited with translation and commentary, Philadelphie (Penns.) $\quad\left[{ }^{1} 1941\right.$ (American Philological Association Monographs, 10)], revised edition with the collaboration of Marcello Gigante, Francesca Longo Auricchio, Adele Tepedino Guerra, Naples, 1978 (La scuola di Epicuro, 1).

GiUsSANI, C. 1896-1898 : T. Lucreti Cari De rerum natura libri sex, revisione del testo, commento e studi introduttivi, 4 vol., Turin, 1896-1898 (Collezione di classici greci e latini con note italiane).

Glidden, D. K. 1973 : « Epicurean Prolepsis », Oxford Studies in Ancient Philosophy, 3 (1985), p. 175-217.

JÜRSS, Fr. 1977 : «Epikur und das Problem des Begriffes (Prolepse) », Philologus, 121 (1977), p. 211-225.

- 1991 : Die epikureische Erkenntnistheorie, Berlin, 1991 (Schriften zur Geschichte und Kultur der Antike, 33).

LONG, A. A. 1971 : « Aisthèsis, Prolepsis and Linguistic Theory in Epicurus », Bulletin of the Institute of Classical Studies of the University of London, 18 (1971), p. 114-133.

LONG, A. A. \& D. SEDley 2001 : Les philosophes hellénistiques.1, Pyrrhon; L'épicurisme, Paris, 2001 (GF, 641) [traduction, par J. Brunschwig et P. Pellegrin, de : The Hellenistic Philosophers, Cambridge, 1987].

Manuwald, A. 1972: Die Prolepsislehre Epikurs, Bonn, 1972 (Habelts Dissertationsdrücke. Klassische Philologie, 15).

MOREL, P.-M. 2002 : «Les ambiguïtés de la conception épicurienne du temps », Revue philosophique de la France et de l'étranger, 192.2 (avril-juin 2002), p. 195-211.

Sedley, D. 1973: «Epicurus, On Nature, Book XXVIII », Cronache Ercolanesi, 3 (1973), p. 5-83.

VERDE, F. 2008 : «Rebus ab ipsis consequitur sensus : il tempo in Epicuro », Elenchos, 29.1 (2008), p. 91-117. 